\title{
Backward-iteration sequences with bounded hyperbolic steps for analytic self-maps of the disk
}

Pietro Poggi-Corradini

\begin{abstract}
A lot is known about the forward iterates of an analytic function which is bounded by 1 in modulus on the unit disk $\mathbb{D}$. The DenjoyWolff Theorem describes their convergence properties and several authors, from the 1880's to the 1980's, have provided conjugations which yield very precise descriptions of the dynamics. Backwarditeration sequences are of a different nature because a point could have infinitely many preimages as well as none. However, if we insist in choosing preimages that are at a finite hyperbolic distance each time, we obtain sequences which have many similarities with the forward-iteration sequences, and which also reveal more information about the map itself. In this note we try to present a complete study of backward-iteration sequences with bounded hyperbolic steps for analytic self-maps of the disk.
\end{abstract}

\section{Introduction}

Let $\phi$ be an analytic self-map of $\mathbb{D}$. The Theorem of Denjoy-Wolff says that, aside for the case when $\phi$ is an elliptic automorphism, there is a point $\tau_{\phi} \in \overline{\mathbb{D}}$ (which we call the Denjoy-Wolff point of $\phi$ ) such that the iterates of $\phi$ converge to $\tau_{\phi}$ uniformly on compact subsets of $\mathbb{D}$. A lot is known about the behavior of a forward-iteration sequence under $\phi, z_{n}=\phi_{n}\left(z_{0}\right)$, especially in the limit, for large values of $n$. In fact, conjugations of $\phi$ to linear maps have been established near the Denjoy-Wolff point and these provide a fairly clear picture of the dynamics. The different types of behavior divide the class of self-maps of the disk into three main categories:

2000 Mathematics Subject Classification: 30D05, 30D50, 39B32.

Keywords: Backward-iteration, bounded steps. 
Elliptic. The Denjoy-Wolff point is an interior fixed point for $\phi$, i.e. $\tau_{\phi} \in \mathbb{D}$. By Schwarz's lemma, the derivative $\lambda$ of $\phi$ at $\tau_{\phi}$ (also known as the multiplier) satisfies $|\lambda| \leq 1$. If $|\lambda|=1, \phi$ is an elliptic automorphism. If $0<|\lambda|<1$, $\phi(z)$ can be conjugated to $\lambda z$ near $\tau_{\phi}$, in such a way that the forward orbit $z_{n}$ is asymptotic to $\lambda^{n} \omega$ for some $\omega$, for large $n$. If $\lambda=0, \phi(z)$ can be conjugated to $z^{N}$ near $\tau_{\phi}$, for some $N \geq 2$, and related asymptotics can be obtained.

Hyperbolic. The Denjoy-Wolff point is a boundary fix point for $\phi$, i.e., $\tau_{\phi} \in \partial \mathbb{D}$ and $\phi\left(\tau_{\phi}\right)=\tau_{\phi}$ in the sense of non-tangential limits. In this case, the derivative $c$ of $\phi$ at $\tau_{\phi}$ exists, again in the sense of non-tangential limits, and satisfies $0<c \leq 1$. The map $\phi$ is hyperbolic if $c<1$. Conjugations exist in this case and can be used to show that $\operatorname{Arg}\left(z_{n}-\tau_{\phi}\right)-\operatorname{Arg} \tau_{\phi}$ tends to an angle $\theta_{0} \in\left(-\frac{\pi}{2}, \frac{\pi}{2}\right)$. In particular, a forward-iteration sequence eventually tends to $\tau_{\phi}$ along a non-tangential ray at $\tau_{\phi}$ (combine [CM, Lemma 2.66] with [Pom, Th. 3(i)]).

Parabolic. The Denjoy-Wolff point $\tau_{\phi} \in \partial \mathbb{D}$ is a boundary fixed point for $\phi$, and the derivative $c$ of $\phi$ at $\tau_{\phi}$ is equal to 1 . This case is more subtle and the behavior of forward-iteration sequences has been studied by Pommerenke and Baker, $[\mathrm{Pom}],[\mathrm{BP}]$, and by Cowen, $[\mathrm{CM}]$. Conjugations still exist: they send $\tau_{\phi}$ to $\infty$ and conjugate $\phi(z)$ to a translation, see Section 1.3 for more details.

Let $d$ be the pseudo-hyperbolic distance in $\mathbb{D}$, i.e., $d(z, w)=\frac{|z-w|}{|1-\bar{z} w|}$. By Schwarz-Pick, forward-iteration sequences always have bounded steps in the hyperbolic metric. More specifically, $d\left(z_{n+1}, z_{n}\right) \leq d\left(z_{n}, z_{n-1}\right)$, and this is maybe the reason why these sequences become more and more "regular" in the limit. Actually, the sequence of step-lengths, $s_{n}=d\left(z_{n+1}, z_{n}\right)$, plays an important role. Clearly $s_{n}$ tends to $s_{\infty} \geq 0$. In the elliptic case $s_{\infty}=0$ (aside for elliptic automorphisms). In the hyperbolic case, $s_{\infty}>0$. More importantly, in the parabolic case, both $s_{\infty}=0$ and $s_{\infty}>0$ can occur, and the dynamics is different in each case. As a matter of notation, we say $\phi$ is type I parabolic if $s_{\infty}>0$, and type II parabolic if $s_{\infty}=0$.

\subsection{Boundary repelling fixed points}

We saw above that $\tau_{\phi}$ is always a fixed point for $\phi$ and the multiplier there is less or equal to 1 in modulus. The Denjoy-Wolff Theorem says, moreover, that every other fixed point of $\phi$ can only be on the boundary of $\mathbb{D}$, with positive multiplier $>1$ (infinity an admitted value). An analytic self-map of the disk may have several such fixed points other than the Denjoy-Wolff point, however, when the multiplier is also finite then more can be said and these points have several nice features. That is the reason why in [PC2] we gave them the name of Boundary Repelling Fixed Points (BRFP). Below are three results involving boundary fixed points which will be useful in the sequel. 
Theorem 1.1 (Julia and Carathéodory, [Sh, Chap. 4]) Suppose $\phi$ is an analytic map of the disk with $\phi(\mathbb{D}) \subset \mathbb{D}$, and $\zeta, \xi \in \partial \mathbb{D}$. If there is a sequence $\left\{p_{n}\right\} \subset \mathbb{D}$ such that $p_{n} \rightarrow \zeta, \phi\left(p_{n}\right) \rightarrow \xi$, and

$$
\frac{1-\left|\phi\left(p_{n}\right)\right|}{1-\left|p_{n}\right|} \rightarrow A<\infty,
$$

then

(a) $A>0$

(b) For every horodisk $H$ at $\zeta$, i.e., $H$ is a disk internally tangent to $\partial \mathbb{D}$ at $\zeta, \phi(H) \subset M(H)$, where $M(z)=\xi \bar{\zeta}(z-a \zeta) /(1-a \bar{\zeta} z)$, with $a=\frac{A-1}{A+1}$.

(c) $\phi(z) \rightarrow \xi$ as $z \rightarrow \zeta$ non-tangentially.

(d) $\phi^{\prime}(z) \rightarrow \phi^{\prime}(\zeta)$ as $z \rightarrow \zeta$ non-tangentially, and $\left|\phi^{\prime}(\zeta)\right| \leq A$.

Corollary 1.2 Suppose $\phi$ is an analytic map with $\phi(\mathbb{D}) \subset \mathbb{D}$, and suppose $\zeta \in \partial \mathbb{D}$. Assume further that there is a sequence $\left\{p_{n}\right\} \subset \mathbb{D}$ such that

1. $p_{n} \rightarrow \zeta$,

2. $\lim _{n \rightarrow \infty} d\left(p_{n}, \phi\left(p_{n}\right)\right) \leq a<1$.

Then $\zeta$ is a boundary fixed point of $\phi$ with multiplier $\phi^{\prime}(\zeta) \leq \frac{1+a}{1-a}$.

Theorem 1.3 (Cowen-Pommerenke, [CP, Theorem 4.1]) Let $\phi$ be analytic with $\phi(\mathbb{D}) \subset \mathbb{D}$, and let $\tau_{\phi}$ be its Denjoy-Wolff point. If $\tau_{\phi} \in \mathbb{D}$ or if $\tau_{\phi} \in \partial \mathbb{D}$ and $\phi^{\prime}\left(\tau_{\phi}\right)<1$ (elliptic and hyperbolic cases), then for every $A>1$ the set of BRFPs whose multiplier is less than $A$ must be finite. Moreover if $\tau_{\phi} \in \partial \mathbb{D}$ and $\phi^{\prime}\left(\tau_{\phi}\right)=1$ (parabolic case), then for every $A>1$ the set of BRFPs whose multiplier is less than $A$ can only cluster at $\tau_{\phi}$.

The two theorems just cited were used in [PC1] to prove the following conjugation result.

Theorem 1.4 ([PC1, Thm. 1.2]) Suppose $\phi$ is analytic with $\phi(\mathbb{D}) \subset \mathbb{D}$. Assume that 1 is a BRFP for $\phi$ with multiplier $1<A<\infty$.

Then there is an analytic map $\psi$ of the upper half-plane $\mathbb{H}$, with $\psi(\mathbb{H}) \subset \mathbb{D}$, which has non-tangential limit 1 at 0 , and such that:

$$
\psi(A z)=\phi \circ \psi(z)
$$

for every $z \in \mathbb{H}$.

Moreover, $\psi$ is always semi-conformal at 0 , that is,

$$
\operatorname{Arg} \frac{\psi(z)-1}{i z} \longrightarrow 0
$$

as z tends to 0 non-tangentially. 
The main construction in the proof of Theorem 1.4 was to produce a backward-iteration sequence with certain additional properties, in particular whose steps remain bounded in the hyperbolic distance.

Definition 1.5 A sequence $\left\{w_{n}\right\}_{0}^{\infty}$ is a backward-iteration sequence with bounded steps (BISBS) for $\phi$, if $\phi\left(w_{n+1}\right)=w_{n}$ for $n=0,1,2,3 \ldots$, and $d\left(w_{n}, w_{n+1}\right) \leq a<1$ for all $n$ and for some constant $a$. (We exclude the trivial sequence $w_{n} \equiv \tau_{\phi}$, in the elliptic case, from this definition, and we also assume that $\phi$ is not an elliptic automorphism in the rest of this paper.)

On the other hand, Theorem 1.4 implies that if $\phi$ has a BRFP at $\zeta$ (say $\zeta=1)$ then there are a lot of BISBS, namely all the sequences of the form $\left\{\psi\left(A^{-n} z\right)\right\}_{n=0}^{\infty}$ for some $z \in \mathbb{H}$.

In this note, we propose to study the BISBS of a self-map $\phi$ of the disk, their convergence properties, as well as existence and uniqueness properties. To begin with, in the result stated in the next section we show that a BISBS can only arise as in Theorem 1.4 above, i.e. tends to a BRFP non-tangentially and is of the form $\left\{\psi\left(A^{-n} z\right)\right\}_{n=0}^{\infty}$ for a conjugation as in Theorem 1.4, except for the parabolic case, where it can happen that a BISBS actually tends to the Denjoy-Wolff point. The parabolic case is more delicate and is the main focus of this paper, see Paragraphs 1.3-1.7 below.

\subsection{Backward-iteration sequences with bounded steps}

We will see that the bounded-steps restriction yields several interesting properties and that these sequences become "regular" for large values of $n$, analogously to the forward-iteration sequences. Note that Schwarz-Pick implies that $d\left(w_{n}, w_{n+1}\right)$ is increasing with $n$, thus without loss of generality we always assume that $d\left(w_{n}, w_{n+1}\right) \uparrow a<1$.

Theorem 1.6 Suppose $\phi$ is an analytic map with $\phi(\mathbb{D}) \subset \mathbb{D}$, and let $\left\{w_{n}\right\}_{n=0}^{\infty}$ be a backward-iteration sequence for $\phi$ with bounded pseudo-hyperbolic steps $d_{n}=d\left(w_{n}, w_{n+1}\right) \uparrow a<1$. Then, the following hold:

1. There is a point $\zeta \in \partial \mathbb{D}$ such that $w_{n} \rightarrow \zeta$ as $n$ tends to infinity, and $\zeta$ is a fixed point for $\phi$ with a well-defined multiplier $\phi^{\prime}(\zeta)<\infty$.

2. When $\zeta \neq \tau_{\phi}$, then $\zeta$ is a BRFP. If $\zeta=\tau_{\phi}$, then $\phi$ is necessarily of parabolic type.

3. When $\zeta$ is a BRFP, the sequence $w_{n}$ tends to $\zeta$ along a non-tangential direction. More precisely, there exists an angle $\theta_{0}$ in $\left(-\frac{\pi}{2}, \frac{\pi}{2}\right)$ such that

$$
\arg \left(\zeta-w_{n}\right)-\arg \zeta \rightarrow \theta_{0} \quad \text { as } n \rightarrow \infty .
$$

4. When, in the parabolic case, $\zeta=\tau_{\phi}$, then $w_{n}$ tends to $\zeta$ tangentially. 
As we have seen above, a consequence of Theorem 1.4 is that whenever $\zeta \in \partial \mathbb{D}$ is a BRFP for $\phi$ then one can construct many BISBS converging to $\zeta$ along non-tangential directions as in (1.4). One of the consequences of Theorem 1.6 (3) is that every BISBS approaching a BRFP must do so non-tangentially as in (1.4). Finally, as mentioned in (2), in the parabolic case it can happen that a BISBS tends to $\tau_{\phi}(=1)$, and we can construct examples in both the type I and type II cases, see Section 4. Theorem 1.6 is proved in Section 2. The rest of the paper is devoted to studying what happens in the parabolic case.

\subsection{Parabolic forward iteration}

We study the parabolic case in Section 3, where we obtain counterparts to results about forward iterates of Pommerenke and Baker-Pommerenke, [Pom] [BP]. First let us recall what is known about forward-iteration in this case. A self-map of $\mathbb{D}$ is parabolic if $\tau_{\phi} \in \partial \mathbb{D}$ and $\phi^{\prime}\left(\tau_{\phi}\right)=1$ there. Without loss of generality, we assume instead that $\phi$ is an analytic map on the upper half-plane $\mathbb{H}$, with $\operatorname{Im} \phi(z) \geq 0$, such that

$$
\phi(z) \longrightarrow \infty \quad \text { and } \quad \frac{\phi(z)}{z} \longrightarrow 1 .
$$

as $z \rightarrow \infty$ non-tangentially. The non-tangential approach regions for $\infty$ in $\mathbb{H}$ are the sectors $\left\{|\operatorname{Arg} z-\pi / 2|<\theta_{0}\right\}$ for some $\theta_{0} \in(0, \pi / 2)$. Since the horodisks at infinity are the half-planes $\{\operatorname{Im} z \geq t>0\}$, Julia's Lemma in this situation implies that $\operatorname{Im} \phi(z) \geq \operatorname{Im} z$. In $\mathbb{H}$ the pseudo-hyperbolic distance between two points $z, w$ is:

$$
d=d(z, w)=\left|\frac{w-z}{w-\bar{z}}\right|
$$

Let $z_{n}=\phi_{n}(i)$. We know that $z_{n}$ tends to infinity by the Denjoy-Wolff Theorem. Also the step-lengths $s_{n}=d\left(z_{n}, z_{n+1}\right)$ decrease to $s_{\infty}$, and $\phi$ is said to be of type I (or non-zero-step) if $s_{\infty}>0 ; \phi$ is of type II (or zero-step) if $s_{\infty}=0$. We will refine the type I and type II classes. By Julia's Lemma, $\operatorname{Im} z_{n} \uparrow L_{\infty}$. A map $\phi$ of type I is said to be of type Ia (or non-zerostep/finite-height) if $L_{\infty}<\infty$ and of type Ib (or non-zero-step/infiniteheight) if $L_{\infty}=\infty$. Likewise for type II.

Example 1.7 The map $\phi(z)=z+1$ is of type Ia, while $\phi(z)=z+i$ is of type IIb. We give more examples in Section 4.

It is not clear a priori that this classification does not depend on the choice of $i$ as starting point, however, that is indeed the case, as we will see below, as a consequence of the next theorem. 
Theorem 1.8 (Pommerenke, [Pom, (3.17)]) Let $\phi$ be an analytic selfmap of $\mathbb{H}$ of parabolic type as in (1.5), and let $\left\{z_{n}=\phi_{n}(i)\right\}_{n=0}^{\infty}$ be a forwarditeration sequence. Then

$$
\frac{\operatorname{Im} z_{n+1}}{\operatorname{Im} z_{n}} \longrightarrow 1
$$

as $n$ tends to infinity.

Moreover, letting $z_{n}=u_{n}+i v_{n}$ and considering the automorphisms of $\mathbb{H}$ given by $M_{n}(z)=\left(z-u_{n}\right) / v_{n}$, the normalized iterates $M_{n} \circ \phi_{n}$ converge uniformly on compact subsets of $\mathbb{H}$ to a function $\sigma$ which satisfies the functional equation

$$
\sigma \circ \phi=\sigma+b
$$

where

$$
b=\lim _{n \rightarrow \infty} \frac{u_{n+1}-u_{n}}{v_{n}}
$$

and $b \neq 0$ in the non-zero-step case, while $b=0$ in the zero-step case.

Since

$$
M_{n} \circ M_{n+1}^{-1}(z)=z \frac{v_{n+1}}{v_{n}}+\frac{u_{n+1}-u_{n}}{v_{n}} \rightarrow z+b,
$$

given an arbitrary point $z \in \mathbb{H}$,

$$
\begin{aligned}
d\left(\phi_{n}(z), \phi_{n+1}(z)\right) & =d\left(M_{n} \circ \phi_{n}(z), M_{n} \circ M_{n+1}^{-1} \circ M_{n+1} \circ \phi_{n+1}(z)\right) \\
& \rightarrow d(\sigma(z), \sigma(z)+b) .
\end{aligned}
$$

Therefore the type I and type II classification is well-defined. The fact that the type a and type b classification is well-defined follows easily from Schwarz-Pick, because $d\left(\phi_{n}(z), \phi_{n}(i)\right) \leq d(z, i)$.

\subsection{Parabolic backward iteration}

We now study parabolic maps $\phi$ as in (1.5) which have a BISBS tending to infinity. The map $\phi(z)=z+i$ shows that such sequences may not exist at all. We continue our classification by saying that $\phi$ is type $\emptyset$ if it has no such BISBS. So, $\phi(z)=z+i$ is of type $\emptyset$. On the other hand, if a BISBS exists $\left\{w_{n}\right\}_{n=0}^{\infty}$, then by Julia's Lemma, $y_{n}:=\operatorname{Im}\left(w_{n}\right) \downarrow \ell_{\infty}$. We say that a BISBS is of type 1 (or non-zero-height) if $\ell_{\infty}>0$, and of type $\mathbf{2}$ (or zero-height) if $\ell_{\infty}=0$. So, for instance, $\phi(z)=z+1$ has a BISBS of type 1 . At first sight, one might think that type 2 never arises. However, a simple example is given by the following map $\phi(z)=\sqrt{z^{2}-1}$. Thinking of $\phi$ as the composition of three simple operations, one checks that $\phi$ maps $\mathbb{H}$ into itself and that it is of parabolic type IIb with Denjoy-Wolff point at infinity. The sequence 
$w_{n}=\sqrt{n+i}$ is a backward-iteration sequence for $\phi$. A calculation shows that the pseudo-hyperbolic steps $d_{n}$ stay bounded away from 1 . So $w_{n}$ is a BISBS and $\operatorname{Im} w_{n} \asymp 1 / \sqrt{n}$ tends to zero. In this example, although $y_{n}$ tends to zero, it does not do so very fast, e.g., $\sum y_{n}=\infty$. This is a general fact.

Lemma 1.9 Let $\phi$ be a parabolic map of $\mathbb{H}$ as above, and let $w_{n}$ be a BISBS tending to infinity. If $y_{n}=\operatorname{Im} w_{n}$, then

$$
\sum_{n=0}^{\infty} y_{n}=\infty
$$

Proof. Recall that $d_{n}=d\left(w_{n}, w_{n+1}\right) \rightarrow a<1$. Then

$$
\frac{1}{d_{n}}=\left|1-\frac{2 y_{n} i}{w_{n+1}-w_{n}}\right| \leq 1+2 \frac{y_{n}}{\left|w_{n+1}-w_{n}\right|} .
$$

So,

$$
\liminf _{n \rightarrow \infty} \frac{y_{n}}{\left|w_{n+1}-w_{n}\right|} \geq \frac{1-a}{2 a} .
$$

Letting $C_{0}=(1-a) / 4 a$, there is $n_{0}$ such that for $n \geq n_{0}, y_{n} \geq C_{0}\left|w_{n+1}-w_{n}\right|$. Summing from $n_{0}$ to $N>n_{0}$,

$$
\begin{aligned}
\sum_{n=n_{0}}^{N} y_{n} & \geq C_{0} \sum_{n=n_{0}}^{N}\left|w_{n+1}-w_{n}\right| \geq C_{0}\left|\sum_{n=n_{0}}^{N}\left(w_{n+1}-w_{n}\right)\right| \\
& =C_{0}\left|w_{N+1}-w_{n_{0}}\right| \rightarrow \infty
\end{aligned}
$$

A main question in this context is whether the ratios $y_{n+1} / y_{n}$ tend to 1 or not. All we can infer so far, from Lemma 1.9 for example, is that

$$
\limsup _{n \rightarrow \infty} \frac{y_{n+1}}{y_{n}}=1 \text {. }
$$

We will show that indeed $\lim _{n \rightarrow \infty} y_{n+1} / y_{n}=1$. It will be useful, in the course of the proof, to consider the hyperbolic steps of higher order. Namely, if $w_{n}$ is a BISBS, and $k=1,2,3, \ldots$, then

$$
d\left(w_{n}, w_{n+k}\right) \uparrow a_{k} .
$$

To see that the $a_{k}$ are also strictly less than one, consider the hyperbolic distance

$$
\rho=\log \frac{1+d}{1-d}
$$

Then $\rho\left(w_{n}, w_{n+k}\right) \uparrow \rho_{k}$, and by the triangle inequality, $\rho_{k} \leq k \rho_{1}<\infty$. Thus, $a_{k}<1$. 
Remark 1.10 Note that $\operatorname{Arg}\left(w_{n}\right)$ either tends to 0 or to $\pi$. In fact, $0<$ $\operatorname{Im} w_{n} \leq \operatorname{Im} w_{0}$ and $w_{n} \rightarrow \infty$. So, if $\operatorname{Arg} w_{n}$ were to oscillate between 0 and $\pi$, the sequence $w_{n}$ would accumulate everywhere on the real axis, because of the bounded-steps condition, and this would yield a contradiction.

The following theorem is a direct counter-part to Theorem 1.8.

Theorem 1.11 Let $\phi$ be an analytic self-map of $\mathbb{H}$ of parabolic type as in (1.5), and let $\left\{w_{n}\right\}_{n=0}^{\infty}$ be a backward-iteration sequence with bounded pseudohyperbolic steps $d_{n}=d\left(w_{n}, w_{n+1}\right) \uparrow a<1$, which tends to infinity. Then

$$
\frac{\operatorname{Im} w_{n+1}}{\operatorname{Im} w_{n}} \longrightarrow 1
$$

as $n$ tends to infinity.

This is the key to proving the following conjugation result.

Theorem 1.12 Let $\phi$ be an analytic self-map of $\mathbb{H}$ of parabolic type as in (1.5), and let $\left\{w_{n}=x_{n}+i y_{n}\right\}_{n=0}^{\infty}$ be a backward-iteration sequence with bounded pseudo-hyperbolic steps $d_{n}=d\left(w_{n}, w_{n+1}\right) \uparrow a<1$, which tends to infinity. (Assume also WLOG that $\operatorname{Arg} w_{n}$ tends to 0). Consider the automorphisms of $\mathbb{H}$ given by $\tau_{n}(z)=x_{n}+y_{n} z$. Then the normalized iterates $\phi_{n} \circ \tau_{n}$ converge uniformly on compact subsets of $\mathbb{H}$ to an analytic self-map $\psi$ of $\mathbb{H}$ such that

$$
\psi\left(z-b_{0}\right)=\phi \circ \psi(z)
$$

where

$$
b_{0}=\frac{2 a}{\sqrt{1-a^{2}}}=\lim _{n \rightarrow \infty} \frac{x_{n+1}-x_{n}}{y_{n}}
$$

Corollary 1.13 With the hypothesis of Theorem 1.11 and Theorem 1.12, letting $\zeta_{n}=i+n b_{0}$,

1. $\psi\left(\zeta_{n}\right)=w_{n}$ and $\psi^{\prime}\left(\zeta_{n}\right) / y_{n} \rightarrow 1$.

2. $\psi$ has non-tangential limit $\infty$ at $\infty$.

3. $\lim \phi^{\prime}\left(w_{n}\right)=1$.

Theorem 1.11, Theorem 1.12 and Corollary 1.13 are proved in Section 3.1. 


\subsection{Uniqueness for BISBS of type 1}

Let $w_{n}$ be a BISBS of non-zero-height as defined in Section 1.4. If $\tau_{n}(z)=$ $x_{n}+z y_{n}$, then Theorem 1.12 says that $\phi_{n} \circ \tau_{n}$ tends to the conjugating map $\psi$. So, given an arbitrary $z \in \mathbb{H}$, the sequence $\left\{\psi\left(z+n b_{0}\right)\right\}_{n=0}^{\infty}$ is a BISBS for $\phi$ and is of non-zero-height as well, by Proposition 1.16 below and by Julia's Lemma. We show that every BISBS of non-zero-height occurs this way.

Theorem 1.14 Let $\phi$ be an analytic self-map of $\mathbb{H}$ of parabolic type, and let $\left\{w_{n}=x_{n}+i y_{n}\right\}_{n=0}^{\infty}$ be a backward-iteration sequence tending to the Denjoy-Wolff point, $\tau_{\phi}=\infty$, with bounded pseudo-hyperbolic steps $d_{n}=$ $d\left(w_{n}, w_{n+1}\right) \uparrow a<1$, and which is furthermore of non-zero-height, i.e., $y_{n} \downarrow \ell_{\infty}>0$. Let $\tilde{w}_{n}$ be another backward-iteration sequence tending to infinity with $d\left(\tilde{w}_{n}, \tilde{w}_{n+1}\right) \uparrow \tilde{a}<1$, and $\tilde{y}_{n} \downarrow \tilde{\ell}_{\infty}>0$. Assume further that $\operatorname{Arg} w_{n}$ and $\operatorname{Arg} \tilde{w}_{n}$ are both tending to zero. Likewise let $\tau_{n}, \tilde{\tau}_{n}, \psi$, and $\tilde{\psi}$, $b_{0}$, and $\tilde{b}_{0}$ be the corresponding maps given by Theorem 1.12. Then

$$
\tilde{\psi}\left(\frac{\tilde{b}_{0}}{b_{0}} z+b_{1}\right)=\psi(z)
$$

for some $b_{1} \in \mathbb{R}$. So that, $\tilde{w}_{n}=\psi\left(z_{1}+n b_{0}\right)$ for $z_{1}=\left(b_{0} / \tilde{b}_{0}\right)\left(i-b_{1}\right)$, and

$$
\lim _{n \rightarrow \infty} x_{n+1}-x_{n}=\ell_{\infty} \frac{2 a}{\sqrt{1-a^{2}}}=\tilde{\ell}_{\infty} \frac{2 \tilde{a}}{\sqrt{1-\tilde{a}^{2}}}=\lim _{n \rightarrow \infty} \tilde{x}_{n+1}-\tilde{x}_{n}
$$

Question 1.15 It is not clear whether Theorem 1.14 holds in the zero-height case, and whether zero-height BISBS can coexist with non-zero-height one. We conjecture that at least for univalent maps $\phi$ this never happens. Theorem 1.21 below implies that this cannot happen for type I parabolic maps.

Theorem 1.14 is proved in Section 3.2.

\subsection{More on BISBS of type 1}

Proposition 1.16 Let $w_{n}=x_{n}+i y_{n}$ be a BISBS of non-zero-height, i.e. such that $y_{n} \downarrow \ell_{\infty}>0$, and assume $x_{n} \rightarrow+\infty$. Let $\psi$ be the conjugating map obtained in Theorem 1.12. Then

$$
\frac{\psi(z)}{z} \longrightarrow \ell_{\infty}
$$

as z tends to infinity non-tangentially.

Corollary 1.17 Let $\psi$ be as in Proposition 1.16, and consider the quadrants $Q(R)=\{x>R, y>1\}$. Then $\psi$ is one-to-one on $Q(R)$ for $R$ large enough, and this is true of $\phi$ as well. 
In the course of the proof of Theorem 1.14 above we found useful to introduce the following notion of limit. For $R>0$ and $\epsilon>0$ define the horizontal half-strips

$$
\mathbb{S}(R, \epsilon)=\{z=x+i y: x \geq R, \epsilon \leq y \leq 1 / \epsilon\} .
$$

Definition 1.18 Given a complex-valued function $f$ defined on $\mathbb{H}$, we say that $f$ has a lateral-limit at $+\infty$ if there is $a \in \mathbb{C}$ such that

$$
\lim _{\mathbb{S}(R, \epsilon) \ni z \rightarrow \infty} f(z)=a
$$

for every choice of $R>0$ and $0<\epsilon<1$. In this case we write,

$$
\text { lat. } \lim _{z \rightarrow+\infty} f(z)=a
$$

Proposition 1.19 Let $\phi$ be an analytic self-map of $\mathbb{H}$ of parabolic type as in (1.5), and let $\left\{w_{n}=x_{n}+i y_{n}\right\}_{n=0}^{\infty}$ be a backward-iteration sequence with bounded pseudo-hyperbolic steps $d_{n}=d\left(w_{n}, w_{n+1}\right) \uparrow a<1$, which tends to infinity, and which is furthermore of non-zero-height, i.e., such that $y_{n} \downarrow$ $\ell_{\infty}>0$. Assume also that $x_{n}$ tends to $+\infty$ and let $b_{0}$ be defined as in (1.9). Then,

$$
\text { lat. }-\lim _{z \rightarrow+\infty} z-\phi(z)=b_{0} \ell_{\infty}
$$

The following question is therefore quite natural, also in view of Theorem 1.4 in the case of a BRFP. In that case, if $\phi$ fixes a point $\zeta \neq \tau_{\phi}$ on the boundary of the unit disk, then the existence of a finite angular derivative at $\zeta$ implies the existence of BISBS tending to $\zeta$.

Question 1.20 Suppose $\phi$ is an analytic self-map of $\mathbb{H}$, as in (1.5). Suppose moreover that

$$
\text { lat. }-\lim _{z \rightarrow+\infty} z-\phi(z)=C_{0}>0
$$

Does this imply that there exists a BISBS of type 1 tending to $+\infty$ ?

Proposition 1.16, Corollary 1.17, and Proposition 1.19 are proved in Section 3.3.

\subsection{BISBS for type I parabolic maps}

We show that if a self-map $\phi$ of $\mathbb{H}$ is of parabolic type I, then it cannot have a BISBS of zero-height.

Theorem 1.21 Let $\phi$ be an analytic self-map of $\mathbb{H}$, as in (1.5), which is of parabolic type I, i.e., the forward iterates have non-zero step. Assume, also that $\phi$ admits a backward-iteration sequence $\left\{w_{n}\right\}_{n=0}^{\infty}$ tending to infinity, with bounded pseudo-hyperbolic steps $d_{n}=d\left(w_{n}, w_{n+1}\right) \uparrow a<1$. Then $w_{n}$ must be of non-zero-height, i.e., $\operatorname{Im} w_{n} \downarrow \ell_{\infty}>0$. 
Corollary 1.22 A map $\phi$ as in (1.5) of type Ia which has forward iterates whose arguments tend to 0 cannot have a BISBS whose argument also tends to 0.

We don't know whether Corollary 1.22 holds for type Ib and type IIa maps. The fact that for type Ib maps the argument of the forward iterates tends to either 0 or $\pi$ is proved in Remark 1 of [Pom]. Theorem 1.21 and Corollary 1.22 are proved in Section 3.4.

We now start with the proof of the various statements. In the last section of the paper, Section 4, we produce as many examples as we could find of the different behaviors, however, some cases are missing.

\section{Convergence properties}

In this section we prove Theorem 1.6. We begin with the proof of (1), which follows a standard line of argument, however, the idea of using Theorem 1.3 was suggested to us by F. Bracci.

Proof of Theorem 1.6 (1). We treat first the elliptic case, $\tau_{\phi} \in \mathbb{D}$. Since $d\left(w_{n}, \tau_{\phi}\right) \leq d\left(w_{n+1}, \tau_{\phi}\right), r=\lim _{n \rightarrow \infty} d\left(w_{n}, \tau_{\phi}\right)$ exists. If $r=0$, then $w_{n}=\tau_{\phi}$ for all $n$ 's. If $0<r<1$, either $\phi$ is an elliptic rotation or there exists a constant $m<1$ such that $\phi$ maps the disk $\left\{z \in \mathbb{D}: d\left(z, \tau_{\phi}\right)<r\right\}$ into the disk $\left\{z \in \mathbb{D}: d\left(z, \tau_{\phi}\right)<m r\right\}$, and this yields a contradiction. Therefore, aside for the trivial case when $\phi$ is a conjugate rotation or $w_{n}$ is identically equal to $\tau_{\phi}$, we must have $r=1$. Any subsequence, $w_{n_{k}}$ tending to a point $\zeta \in \partial \mathbb{D}$ satisfies all the hypothesis of Corollary 1.2. Thus $\zeta$ is a BRFP for $\phi$ with multiplier bounded by $A:=(1+a) /(1-a)$. However, the fact that $\left\{w_{n}\right\}$ has bounded steps implies that its cluster set on $\partial \mathbb{D}$ must be connected, and hence is either a point or an interval. To see this, connect $w_{n}$ to $w_{n+1}$ with a straight segment to obtain a curve which has the same cluster set as the sequence $\left\{w_{n}\right\}$ on $\partial \mathbb{D}$. Such cluster set can't be an interval, because by Theorem 1.3, the set of BRFPs is at most countable (actually finite in the elliptic and hyperbolic cases since the multipliers stay bounded). So there is a unique $\zeta \in \partial \mathbb{D}$, such that $w_{n} \rightarrow \zeta$ and $\zeta$ is a BRFP for $\phi$, i.e. $1<\phi^{\prime}(\zeta) \leq A$.

We now treat the hyperbolic case. Without loss of generality, we assume that $\tau_{\phi}=1$ and $0<\phi^{\prime}(1):=c<1$. Recall the Poisson kernel at 1 :

$$
P(w)=\frac{1-|w|^{2}}{|1-w|^{2}}
$$

Horocycles at 1 , i.e. disks interior to $\mathbb{D}$ and tangent to $\partial \mathbb{D}$ at 1 , can also be defined as level sets for $P: H(t)=\{w: P(w)>1 / t\}$. 
The existence of the angular derivative $\phi^{\prime}(1)$ implies, by Julia's Lemma (see [Sh], p. 63), that the horocycle $H(t)$ is mapped into the horocycle $H(c t)$. Choose $t_{0}$ so small that $w_{0}$ does not belong to $H\left(t_{0}\right)$. Then, $w_{n}$ is not in $H\left(c^{-n} t_{0}\right)$, hence the sequence $w_{n}$ cannot have cluster points in $\mathbb{D}$. By the same arguments as in Case 1 , we obtain that either $\left\{w_{n}\right\}$ converges to a BRFP $\zeta$, or $\left\{w_{n}\right\}$ converges to 1 . Theorem 1.6 (2) claims that the latter option is impossible.

Finally, in the parabolic case, assume $\tau_{\phi}=1$ and $\phi^{\prime}(1):=c=1$. Note first that, $\left\{w_{n}\right\}$ cannot cluster in $\mathbb{D}$. If so, there would be a subsequence $w_{n_{k}}$ tending to $z_{0} \in \mathbb{D}$. But Schwarz-Pick implies that

$$
d\left(\phi_{n_{k}}\left(z_{0}\right), w_{0}\right) \leq d\left(z_{0}, w_{n_{k}}\right) \longrightarrow 0
$$

and this contradicts the fact that $\phi_{n_{k}}\left(z_{0}\right)$ tends to 1 by the Denjoy-Wolff Theorem. Again, we conclude as before that either $\left\{w_{n}\right\}$ converges to a BRFP $\zeta$, or $\left\{w_{n}\right\}$ converges to 1 . This time however the latter option can occur. A trivial example is the parabolic automorphism $z \mapsto z+1$ on the upper half-plane (conjugated to the unit disk), but one can find other examples as well, e.g. let $\sigma$ be a Riemann map of $\mathbb{D}$ onto the set $\Omega=\{x+i y$ : $\left.y>\chi_{(-\infty, 0])}(x)\right\}$ which sends 1 to $\infty$, and set $\phi(z)=\sigma^{-1}(\sigma(z)+1)$.

We now prove Theorem 1.6 (2). In view of the Proof of (1), it only remains to show that in the hyperbolic case a BISBS cannot tend to $\tau_{\phi}$. This fact, under the more stringent hypothesis of univalence on $\phi$, was already obtained by $\mathrm{F}$. Bracci in $[\mathrm{Br}]$ by different methods than ours. We thank Prof. Bracci for sharing his preprint with us.

Before tackling the proof of Theorem 1.6 (2), we want to obtain an easy consequence of the bounded steps condition: $d\left(w_{n}, w_{n+1}\right) \leq a<1$. The furthest $w_{n+1}$ can be from the origin is $\left(\left|w_{n}\right|+a\right) /\left(1+a\left|w_{n}\right|\right)$. Therefore,

$$
1-\left|w_{n+1}\right| \geq \frac{1-a}{1+a}\left(1-\left|w_{n}\right|\right) .
$$

On the other hand, the world's-greatest-identity tells us that

$$
\begin{aligned}
1-a^{2} & \leq 1-d\left(w_{n}, w_{n+1}\right)^{2}=\frac{\left(1-\left|w_{n}\right|^{2}\right)\left(1-\left|w_{n+1}\right|^{2}\right)}{\left|1-\overline{w_{n}} w_{n+1}\right|^{2}} \\
& \leq 4 a^{2} \frac{\left(1-\left|w_{n}\right|\right)\left(1-\left|w_{n+1}\right|\right)}{\left|w_{n}-w_{n+1}\right|^{2}} .
\end{aligned}
$$

Putting these two estimates together we obtain

$$
\frac{1-\left|w_{n+1}\right|}{\left|w_{n+1}-w_{n}\right|} \geq \frac{1-a}{2 a}>0 .
$$


Lemma 2.1 Let $\left\{w_{n}\right\}_{n=0}^{\infty} \subset \mathbb{D}$ be a sequence such that $w_{n} \rightarrow \zeta \in \partial \mathbb{D}$ as $n$ tends to infinity. Assume that $w_{n}$ satisfies (2.2) and also

$$
\liminf _{n \rightarrow \infty} \frac{1-\left|w_{n}\right|}{1-\left|w_{n+1}\right|} \geq C>1 .
$$

Then $w_{n}$ converges to $\zeta$ non-tangentially, i.e.,

$$
\liminf _{n \rightarrow \infty} \frac{1-\left|w_{n}\right|}{\left|\zeta-w_{n}\right|} \geq \delta>0
$$

Proof. Note that

$$
\left|w_{n+1}\right|-\left|w_{n}\right|=\left(1-\left|w_{n}\right|\right)-\left(1-\left|w_{n+1}\right|\right)=\left(1-\left|w_{n+1}\right|\right)\left(\frac{1-\left|w_{n}\right|}{1-\left|w_{n+1}\right|}-1\right)
$$

Thus, by (2.3),

$$
\liminf _{n \rightarrow \infty} \frac{\left|w_{n+1}\right|-\left|w_{n}\right|}{1-\left|w_{n+1}\right|} \geq C-1>0
$$

and by (2.2),

$$
\liminf _{n \rightarrow \infty} \frac{\left|w_{n+1}\right|-\left|w_{n}\right|}{\left|w_{n+1}-w_{n}\right|} \geq(C-1) \frac{1-a}{2 a}=\delta>0,
$$

i.e., there is $n_{0}$ such that for $k \geq n_{0}$ :

$$
\left|w_{k+1}\right|-\left|w_{k}\right| \geq \frac{\delta}{2}\left|w_{k+1}-w_{k}\right|
$$

So by telescoping sums, for $m>n>n_{0}$,

$$
\left|w_{m}\right|-\left|w_{n}\right| \geq \frac{\delta}{2}\left|w_{m}-w_{n}\right|
$$

Letting $m$ tend to infinity and then taking the liminf as $n$ tends to infinity, we find that (2.4) holds.

Now we are in position to prove (2) in Theorem 1.6, i.e., we need to show that in the hyperbolic case a backward-iteration sequence with bounded hyperbolic steps cannot tend to the Denjoy-Wolff point.

Proof of Theorem 1.6 (2). Without loss of generality assume that $\tau_{\phi}=1$, so $0<c:=\phi^{\prime}(1)<1$. Also assume that $w_{n}$ does tend to 1 , we will reach a contradiction.

First we rewrite (2.2) as follows

$$
\frac{1-a}{2 a}\left|1-\frac{1-w_{n}}{1-w_{n+1}}\right| \leq \frac{1-\left|w_{n+1}\right|}{\left|1-w_{n+1}\right|}
$$


Julia's Lemma tells us that since $w_{n}$ does not belong to the horocycle $H(t)$ with $t=\left|1-w_{n}\right|^{2} /\left(1-\left|w_{n}\right|^{2}\right), w_{n+1}$ cannot belong to the horocycle $H(t / c)$, i.e.,

$$
\frac{1-\left|w_{n+1}\right|^{2}}{\left|1-w_{n+1}\right|^{2}} \leq c \frac{1-\left|w_{n}\right|^{2}}{\left|1-w_{n}\right|^{2}}
$$

Iterating this estimate we find that

$$
\lim _{n \rightarrow \infty} \frac{1-\left|w_{n}\right|^{2}}{\left|1-w_{n}\right|^{2}}=0
$$

Applying this to (2.5) we obtain that

$$
\lim _{n \rightarrow \infty} \frac{1-w_{n}}{1-w_{n+1}}=1
$$

Going back to (2.6),

$$
\frac{1-\left|w_{n}\right|^{2}}{1-\left|w_{n+1}\right|^{2}} \geq \frac{1}{c}\left|\frac{1-w_{n}}{1-w_{n+1}}\right|
$$

Therefore,

$$
\liminf _{n \rightarrow \infty} \frac{1-\left|w_{n}\right|}{1-\left|w_{n+1}\right|}=\liminf _{n \rightarrow \infty} \frac{1-\left|w_{n}\right|^{2}}{1-\left|w_{n+1}\right|^{2}} \geq \frac{1}{c}>1 .
$$

Now we can apply Lemma 2.1 , with $C$ replaced by $1 / c$, and deduce that $(2.4)$ holds with $\zeta=1$. This contradicts $(2.7)$.

Proof of Theorem 1.6 (3). Suppose now that $w_{n}$ is a BISBS converging to a BRFP $\zeta \in \partial \mathbb{D}$. Without loss of generality $\zeta=1$. Let $A:=\phi^{\prime}(1)>1$. By Theorem 1.1,

$$
A=\liminf _{z \rightarrow \zeta} \frac{1-|\phi(z)|}{1-|z|} .
$$

In particular, $w_{n}$ satisfies (2.3) with $C=A$. Therefore, by Lemma 2.1, we find that (2.4) holds.

Now let $\psi$ be the conjugation produced by Theorem 1.4. In Lemma 5.1 of $[\mathrm{PC} 1]$ we show the existence of a simply connected region $\Omega \subset \mathbb{H}$ with an inner tangent at 0 with respect to $\mathbb{H}$, such that $\psi$ is one-to-one on $\Omega$, and so that $\psi(\Omega)$ has an inner tangent at 1 with respect to $\mathbb{D}$. So, eventually, $w_{n} \in \psi(\Omega)$. Let $f$ be the inverse of $\psi_{\mid \Omega}$. Then $b_{n}:=f\left(w_{n}\right)=A^{-n} b_{0}$ for some $b_{0} \in \mathbb{H}$. By (1.3), this proves (1.4).

Remark 2.2 We found that the proof of Lemma 5.1 of [PC1] has a typo, i.e., $\theta_{n} \uparrow \pi / 2$ instead of $\theta_{n} \downarrow 0$.

Proof of Theorem 1.6 (4). Assume now that $\tau_{\phi}=1$ and $c:=\phi^{\prime}(1)=1$. Assume also that $\left\{w_{n}\right\}_{n=0}^{\infty}$ is a BISBS coverging to 1. By Julia's Lemma, $P\left(w_{n+1}\right) \leq P\left(w_{n}\right)$, where $P$ is defined in (2.1). This shows that $w_{n}$ tends to 1 tangentially. 


\section{The parabolic case}

\subsection{Proof of Theorem 1.11, Theorem 1.12 and Corollary 1.13}

We write $w_{n}=x_{n}+i y_{n}$ for simplicity. Note that if $y_{n} \downarrow \ell_{\infty}>0$, the fact that $y_{n+1} / y_{n} \rightarrow 1$ is clear in this case. However, we will treat both cases together. Recall that $d_{n}=d\left(w_{n}, w_{n+1}\right) \uparrow a<1$ and that $\tau_{n}(z)=x_{n}+z y_{n}$. Notice that

$$
\tau_{n}^{-1} \circ \tau_{n+1}(z)=\frac{x_{n+1}-x_{n}}{y_{n}}+\frac{y_{n+1}}{y_{n}} z
$$

is another automorphism of $\mathbb{H}$, and $d_{n}=d\left(i, \tau_{n}^{-1} \circ \tau_{n+1}(i)\right)$. Hence, we obtain after some manipulation:

$$
\left(\frac{x_{n+1}-x_{n}}{y_{n}}\right)^{2}=-\left(\frac{y_{n+1}}{y_{n}}\right)^{2}+2 \frac{1+d_{n}^{2}}{1-d_{n}^{2}}\left(\frac{y_{n+1}}{y_{n}}\right)-1 .
$$

The right hand-side being a concave-down quadratic polynomial in $y_{n+1} / y_{n}$ implies that

$$
\frac{\left|x_{n+1}-x_{n}\right|}{y_{n}} \leq \frac{2 d_{n}}{1-d_{n}^{2}} \leq \frac{2 a}{1-a^{2}}
$$

and, the left hand-side being positive yields

$$
\frac{1-a}{1+a} \leq \frac{\left(1-d_{n}\right)^{2}}{1-d_{n}^{2}}=\frac{1+d_{n}^{2}}{1-d_{n}^{2}}-\frac{2 d_{n}}{1-d_{n}^{2}} \leq \frac{y_{n+1}}{y_{n}} \leq 1 .
$$

Claim 3.1 The following holds:

$$
f_{n}(z):=\frac{\phi \circ \tau_{n+1}(z)-w_{n}}{\phi \circ \tau_{n+1}(z)-\overline{w_{n}}} \cdot \frac{z+i}{z-i} \longrightarrow 1
$$

as $n$ tends to infinity, uniformly on compact subsets of $\mathbb{H}$.

Proof. Since $\phi\left(\tau_{n+1}(i)\right)=\phi\left(w_{n+1}\right)=w_{n}, f_{n}(z)$ is a well-defined analytic function for $z \in \mathbb{H}$, and by Schwarz-Pick,

$$
\left|f_{n}(z)\right|=\frac{d\left(\phi \circ \tau_{n+1}(z), \phi \circ \tau_{n+1}(i)\right)}{d(z, i)} \leq 1
$$

for all $z \in \mathbb{H}$.

We first show that $\left|f_{n}(z)\right|$ tends to 1 uniformly on compact subsets of $\mathbb{H}$. Consider a subsequence $f_{n_{j}}$. By normal families, we can extract a subsequence $f_{N}$ tending to $f$. By (3.3) and (3.4), we can extract a subsequence $M$ 
of $N$ so that $y_{M+2} / y_{M+1} \rightarrow c \leq 1$ and $\left(x_{M+2}-x_{M+1}\right) / y_{M+1} \rightarrow b \geq 0$ ( $b$ can be chosen to be positive because we can assume without loss of generality that $\operatorname{Arg} w_{n}$ tends to 0 , in view of Remark 1.10). Thus, $\tau_{M+1}^{-1} \circ \tau_{M+2}$ converges uniformly on compact subsets of $\mathbb{C}$ to the automorphism $S(z)=c z+b$, and by $(3.2)$,

$$
b^{2}=-c^{2}+2 \frac{1+a^{2}}{1-a^{2}} c-1
$$

By Schwarz-Pick again,

$$
d\left(\phi \circ \tau_{M+1} \circ S(i), \phi \circ \tau_{M+2}(i)\right) \leq d\left(S(i), \tau_{M+1}^{-1} \circ \tau_{M+2}(i)\right) \rightarrow 0
$$

as $M$ tends to infinity. Using the triangle inequality for the hyperbolic distance, and then transferring it back to the pseudo-hyperbolic distance (this works because of (3.7)), we obtain

$$
\begin{aligned}
\left|f_{M}(S(i))\right| & =\frac{d\left(\phi \circ \tau_{M+1} \circ S(i), \phi \circ \tau_{M+1}(i)\right)}{d(S(i), i)} \\
& \geq \frac{d\left(\phi \circ \tau_{M+2}(i), \phi \circ \tau_{M+1}(i)\right)}{d(S(i), i)}-o(1) \\
& =\frac{d\left(w_{M}, w_{M+1}\right)}{d(S(i), i)}-o(1)
\end{aligned}
$$

On the other hand, using (3.6),

$$
d(S(i), i)^{2}=\frac{b^{2}+(c-1)^{2}}{b^{2}+(c+1)^{2}}=a^{2}
$$

Thus, since $d_{n}$ tends to $a$,

$$
\lim _{M \rightarrow \infty}\left|f_{M}(S(i))\right|=1 .
$$

Thus $f$ is a constant of modulus one, and

$$
\lim _{n \rightarrow \infty}\left|f_{n}(z)\right|=1
$$

uniformly on compact subsets of $\mathbb{H}$.

On the other hand,

$$
f_{n}(i)=\left(\phi \circ \tau_{n+1}\right)^{\prime}(i) \cdot \frac{2 i}{w_{n}-\overline{w_{n}}}=\phi^{\prime}\left(w_{n+1}\right) \cdot \frac{y_{n+1}}{y_{n}}
$$

So

$$
\lim _{n \rightarrow \infty} \frac{y_{n+1}}{y_{n}}\left|\phi^{\prime}\left(w_{n+1}\right)\right|=1 .
$$


Write $\phi(z)=z+p(z)$ with $\operatorname{Im} p(z) \geq 0$ and $p(z) \rightarrow 0$ as $z \rightarrow \infty$ nontangentially. Note that,

$$
\frac{\operatorname{Im} p\left(w_{n+1}\right)}{y_{n+1}}=\frac{\operatorname{Im}\left(w_{n}-w_{n+1}\right)}{y_{n+1}}=\frac{y_{n}}{y_{n+1}}-1
$$

Also Schwarz-Pick applied to $p$ yields,

$$
\frac{y_{n+1}}{y_{n}}\left|p^{\prime}\left(w_{n+1}\right)\right| \leq \frac{y_{n+1}}{y_{n}} \frac{\operatorname{Im} p\left(w_{n+1}\right)}{\operatorname{Im} w_{n+1}}=1-\frac{y_{n+1}}{y_{n}}
$$

for all $n$. Therefore,

$$
\begin{aligned}
\left(\frac{y_{n+1}}{y_{n}}\right)^{2}\left|\phi^{\prime}\left(w_{n+1}\right)\right|^{2} & =\left(\frac{y_{n+1}}{y_{n}}\right)^{2}\left(1+\left|p^{\prime}\left(w_{n+1}\right)\right|^{2}+2 \operatorname{Re} p^{\prime}\left(w_{n+1}\right)\right) \\
& \leq 2 \frac{y_{n+1}}{y_{n}}\left(\frac{y_{n+1}}{y_{n}} \operatorname{Re} \phi^{\prime}\left(w_{n+1}\right)-1\right)+1
\end{aligned}
$$

Rearraging this inequality, using the fact that $\left(y_{n+1} / y_{n}\right) \operatorname{Re} \phi^{\prime}\left(w_{n+1}\right)$ is less than 1 (by (3.5) applied to $z=i$ ), and the fact that $y_{n+1} / y_{n}$ is greater than $(1-a) /(1+a)$, see $(3.4)$, we find that

$$
0 \leq 2 \frac{1-a}{1+a}\left(1-\frac{y_{n+1}}{y_{n}} \operatorname{Re} \phi^{\prime}\left(w_{n+1}\right)\right) \leq 1-\left(\frac{y_{n+1}}{y_{n}}\right)^{2}\left|\phi^{\prime}\left(w_{n+1}\right)\right|^{2}
$$

By (3.10), we obtain

$$
\lim _{n \rightarrow \infty} \frac{y_{n+1}}{y_{n}} \operatorname{Re} \phi^{\prime}\left(w_{n+1}\right)=1 .
$$

Therefore, $f_{n}(i) \rightarrow 1$, and thus $f_{n}(z) \rightarrow 1$ on compact subsets of $\mathbb{H}$. So Claim 3.1 is proved.

Renormalize the iterates of $\phi$ by writing $\psi_{n}(z)=\phi_{n} \circ \tau_{n}(z)$. Note that $\psi_{n}(i)=w_{0}$ and $\operatorname{Im} \psi_{n}>0$. By Claim 3.1,

$$
f_{n}(z)=\frac{\tau_{n}^{-1} \circ \phi \circ \tau_{n+1}(z)-i}{\tau_{n}^{-1} \circ \phi \circ \tau_{n+1}(z)+i} \cdot \frac{z+i}{z-i} \rightarrow 1
$$

which implies

$$
\lim _{n \rightarrow \infty} \tau_{n}^{-1} \circ \phi \circ \tau_{n+1}(z)=z .
$$

So, by Schwarz-Pick applied to $\phi_{n}$ and conformal invariance,

$$
d\left(\psi_{n+1}(z), \psi_{n}(z)\right) \leq d\left(\tau_{n}^{-1} \circ \phi \circ \tau_{n+1}(z), z\right) \rightarrow 0
$$

as $n$ tends to infinity. 
Let $y_{N}$ be a subsequence of the $y_{n}$ such that the ratios $y_{N+1} / y_{N}$ converge to a constant $c$. At the moment, we only know that

$$
0<\frac{1-a}{1+a} \leq c \leq 1
$$

Recall that $\tau_{N}^{-1} \circ \tau_{N+1}$ tends to an automorphism $S(z)=c z+b$ of $\mathbb{H}$, where $b$ is determined by (3.6). By normal families we can assume, passing to a subsequence, that the corresponding sequence of normalized iterates $\psi_{N}$ tends to an analytic function $\psi$ uniformly on compact subsets of $\mathbb{H}$. Then, $\psi(i)=w_{0}$ and $\operatorname{Im} \psi(z)>0$ for all $z \in \mathbb{H}$. Note that

$$
\begin{aligned}
d\left(\psi_{N}(S(i)), w_{1}\right) & =d\left(\phi_{N} \circ \tau_{N} \circ S(i), \phi_{N} \circ \tau_{N+1}(i)\right) \\
& \leq d\left(S(i), \tau_{N}^{-1} \circ \tau_{N+1}(i)\right) \rightarrow 0
\end{aligned}
$$

as $N$ tends to infinity. Thus, $\psi(S(i))=w_{1} \neq w_{0}$, and $\psi$ is not constant. By (3.13), the sequence $\psi_{N+1}$ tends to the same function $\psi$, and since $\psi_{N+1}=$ $\phi \circ \psi_{N} \circ\left(\tau_{N}^{-1} \circ \tau_{N+1}\right)$, we find that $\psi$ must satisfy the functional equation

$$
\psi=\phi \circ \psi \circ S
$$

We now consider the sequence $\psi_{N+2}$, which also tends to $\psi$. Again $\psi_{N+2}=$ $\phi \circ \psi_{N+1} \circ\left(\tau_{N+1}^{-1} \circ \tau_{N+2}\right)$. By the same arguments as before, see (3.3) (3.4) and (3.1), given a subsequence of $N$ we can extract another subsequence, which we call $M$, so that $\tau_{M+1}^{-1} \circ \tau_{M+2}$ converges to an automorphism $\tilde{S}(z)=$ $\tilde{c} z+\tilde{b}$. Then, $\tilde{S}$ satisfies

$$
\phi \circ \psi \circ \tilde{S}=\psi=\phi \circ \psi \circ S .
$$

Since $\phi \circ \psi$ is non-constant we can invert it locally, and since $S$ and $\tilde{S}$ are linear, they must coincide. In particular, $\tau_{N+1}^{-1} \circ \tau_{N+2}$ must converge to $S$ as well. A similar argument yields, for every $k=0,1,2,3 \ldots$, that $\tau_{N+k}^{-1} \circ \tau_{N+k+1}$ tends to $S$. Therefore, $\tau_{N}^{-1} \circ \tau_{N+k}$ must tend to $S_{k}=S \circ \cdots \circ S, k$ times. Write $\zeta_{k}=S_{k}(i)$. Then

$$
d\left(\psi_{N}\left(\zeta_{k}\right), w_{k}\right)=d\left(\phi_{N} \circ \tau_{N}\left(\zeta_{K}\right), \phi_{N} \circ \tau_{N+k}(i)\right) \leq d\left(\zeta_{k}, \tau_{N}^{-1} \circ \tau_{N+k}(i)\right) \rightarrow 0
$$

that is to say

$$
\psi\left(\zeta_{k}\right)=w_{k}
$$

We now consider the functions

$$
g_{n}(z)=\frac{\psi \circ S_{n}(z)-w_{n}}{\psi \circ S_{n}(z)-\overline{w_{n}}} \cdot \frac{z+i}{z-i}
$$


Since $\psi \circ S_{n}(i)=w_{n}$, the $g_{n}$ are analytic on $\mathbb{H}$. Moreover, by Schwarz-Pick,

$$
\left|g_{n}(z)\right|=\frac{d\left(\psi \circ S_{n}(z), \psi \circ S_{n}(i)\right)}{d(z, i)} \leq 1
$$

Note that by (3.8)

$$
\left|g_{n}\left(\zeta_{1}\right)\right|=\frac{d\left(w_{n+1}, w_{n}\right)}{d\left(\zeta_{1}, i\right)} \rightarrow \frac{a}{d\left(\zeta_{1}, i\right)}=1
$$

Therefore, any subsequence of $\left|g_{n}(z)\right|$ has a subsequence converging to a constant of modulus one, i.e.,

$$
\left|g_{n}(z)\right| \longrightarrow 1
$$

as $n$ tends to infinity, for all $z \in \mathbb{H}$. Evaluating $\left|g_{n}\right|$ at $\zeta_{k}$, we obtain

$$
\frac{d\left(w_{n+k}, w_{n}\right)}{d\left(\zeta_{k}, i\right)} \longrightarrow 1
$$

as $n$ tends to infinity. Thus, the number $a_{k}$ introduced in (1.8) satisfy

$$
a_{k}=d\left(\zeta_{k}, i\right)
$$

Recall that $\zeta_{k}=S_{k}(i)$ and $S(z)=c z+b$. Now assume that $c<1$, then

$$
\zeta_{k}=c^{k} i+\frac{1-c^{k}}{1-c} b \longrightarrow \frac{b}{1-c}
$$

Therefore, for $k$ large,

$$
1-a_{k}^{2}=\frac{4 c^{k}}{\left|\zeta_{k}+i\right|^{2}} \leq 4 c^{k}
$$

On the other hand, if $c=1$, then $\zeta_{k}=i+k b_{0}$, where by (3.6),

$$
b_{0}=\frac{2 a}{\sqrt{1-a^{2}}} \text {. }
$$

Thus, for $k$ large,

$$
1-a_{k}^{2}=\frac{4}{\left|\zeta_{k}+1\right|^{2}}=\frac{4}{4+b_{0}^{2} k^{2}} \geq \frac{C}{k^{2}}
$$

for some constant $C>0$. 
These two asymptotic behaviors of the numbers $a_{k}$, as $k$ tends to infinity, show that the sequence of ratios $y_{n+1} / y_{n}$ must converge, and (1.7) implies that the limit must be one. So Theorem 1.11 is proved.

Therefore we have

$$
\tau_{n}^{-1} \circ \tau_{n+1} \rightarrow z+b_{0}:=S(z)
$$

where $b_{0}$ is given in (3.16). Moreover, letting $\psi$ be a normal limit of the $\psi_{n}=\phi_{n} \circ \tau_{n}$, we have have $\psi=\phi_{n} \circ \psi \circ S_{n}$. Also, letting

$$
h_{n}(z)=\tau_{n}^{-1} \circ \psi \circ S_{n}(z)
$$

we find that

$$
h_{n}\left(\zeta_{k}\right)=\tau_{n}^{-1} \circ \tau_{n+k}(i) \rightarrow \zeta_{k}
$$

So $h_{n}$ must tend to the identity, and by the same argument as in [PC1],

$$
\begin{aligned}
d\left(\phi_{n} \circ \tau_{n}, \psi\right) & =d\left(\phi_{n} \circ \tau_{n}, \psi \circ S_{n}^{-1} \circ S_{n}\right) \\
& =d\left(\phi_{n} \circ \tau_{n}, \phi_{n} \circ \psi \circ S_{n}\right) \\
& \leq d\left(\tau_{n}, \psi \circ S_{n}\right) \rightarrow 0 .
\end{aligned}
$$

This proves Theorem 1.12.

Next we show Corollary 1.13. The fact that $\psi\left(\zeta_{n}\right)=w_{n}$ is (3.15). Also, by differentiating $h_{n}(z) \rightarrow z$, see (3.17), we find that

$$
\frac{\psi^{\prime}\left(\zeta_{n}\right)}{y_{n}} \rightarrow 1
$$

as $n$ tends to infinity. This is Corollary $1.13(1)$. In particular, $\psi^{\prime}\left(\zeta_{n}\right) \neq 0$ for $n$ large.

Now consider the half-line $\gamma=\cup_{n=0}^{\infty}\left[\zeta_{n}, \zeta_{n+1}\right]$. Then $\psi$ tends to infinity along $\gamma$ because $\psi\left(\zeta_{n}\right)$ does and for $\zeta \in\left[\zeta_{n}, \zeta_{n+1}\right]$,

$$
d\left(\psi(\zeta), \psi\left(\zeta_{n}\right)\right) \leq \text { Const. }
$$

So by Lindelöff's Theorem, see [Pom2, Cor. 2.17 (i)], $\psi$ has non-tangential limit $\infty$ at $\infty$.

Finally, Corollary 1.13 (3) follows from differentiating (3.12).

\subsection{Uniqueness in the non-zero-height case}

Here we show Theorem 1.14 and so we refer to the assumptions made in the statement. The next Lemma amounts to say that $\psi(z)$ is asymptotic to the map $\ell_{\infty} z$ as $z$ tends to $\infty$ "laterally". For the definition of lat. $-\lim _{z \rightarrow+\infty}$ see Definition 1.18. 
Lemma 3.2 With $\psi$ as in Theorem 1.14, the following holds,

$$
\begin{aligned}
& \text { lat. }-\lim _{z \rightarrow+\infty} \frac{\operatorname{Im} \psi(z)}{\operatorname{Im} z}=\ell_{\infty} \\
& \text { lat. }-\lim _{z \rightarrow+\infty} \frac{\operatorname{Re} \psi(z)}{\operatorname{Re} z}=\ell_{\infty}
\end{aligned}
$$

Moreover, we also have

$$
\text { lat. } \lim _{z \rightarrow+\infty} \psi^{\prime}(z)=\ell_{\infty} \text {. }
$$

Proof. Recall that $y_{n} \rightarrow \ell_{\infty}$ and $x_{n+1}-x_{n} \rightarrow b_{0} \ell_{\infty}$. So given $\varepsilon>0$ there is $n_{0}$ such that for $n \geq n_{0}$

$$
b_{0} \ell_{\infty}-\varepsilon \leq x_{n+1}-x_{n} \leq b_{0} \ell_{\infty}+\varepsilon
$$

So

$$
x_{n_{0}}+\left(n-n_{0}\right)\left(b_{0} \ell_{\infty}-\varepsilon\right) \leq x_{n} \leq x_{n_{0}}+\left(n-n_{0}\right)\left(b_{0} \ell_{\infty}+\varepsilon\right)
$$

Hence

$$
\lim _{n \rightarrow \infty} \frac{x_{n}}{n b_{0}}=\ell_{\infty}
$$

Now recall that $h_{n}(z)=\tau_{n}^{-1} \circ \psi \circ S_{n}(z)$ tends to $z$ uniformly on compact subsets of $\mathbb{H}$, see (3.17). Therefore

$$
\lim _{n \rightarrow \infty}\left|\psi\left(z+n b_{0}\right)-\left(x_{n}+z y_{n}\right)\right|=0
$$

In particular,

$$
\lim _{n \rightarrow \infty}\left|\operatorname{Im} \psi\left(z+n b_{0}\right)-\ell_{\infty} \operatorname{Im} z\right|=0
$$

uniformly for $z \in\left\{0 \leq x \leq b_{0}, \epsilon \leq y \leq 1 / \epsilon\right\}$. This implies (3.18). And also,

$$
\lim _{n \rightarrow \infty}\left|\operatorname{Re} \psi\left(z+n b_{0}\right)-x_{n}-\operatorname{Re} z \ell_{\infty}\right|=0
$$

which if we divide by $\operatorname{Re} z+n b_{0}$ still tends to zero. So, by (3.21),

$$
\lim _{n \rightarrow \infty} \frac{\operatorname{Re} \psi\left(z+n b_{0}\right)}{\operatorname{Re} z+n b_{0}}=\ell_{\infty}
$$

This implies (3.19). Finally, by differentiating $\tau_{n}^{-1} \circ \psi \circ S_{n}(z)$ we find that $\psi^{\prime}\left(z+n b_{0}\right) / y_{n}$ tends to 1 uniformly on compact subsets of $\mathbb{H}$, which implies (3.20). 
As a corollary we obtain the following result which is similar to Lemma 5.1 of [PC1]. First we need another definition.

Definition 3.3 Given a simply connected region $\Omega$ in $\mathbb{H}$, we say that $\Omega$ has an inner-lateral tangent at $+\infty$, if for every choice of $0<\epsilon<1$ there is $R>0$ large enough so that the half-strip $\mathbb{S}(R, \epsilon)$ is contained in $\Omega$.

Corollary 3.4 Let $\psi$ be the conjugation obtained in Theorem 1.12 for a BISBS of type 1 whose argument tends to 0 . Then, there is a convex region $\Omega \subset \mathbb{H}$ with an inner-lateral tangent at $+\infty$, such that $\psi$ is one-to-one on $\Omega$ and $\psi(\Omega)$ also has an inner-lateral tangent at $+\infty$.

Proof. We build $\Omega$ by induction on $k=2,3,4 \ldots$ For $k=2$, choose $R_{2}>0$ so that the three limits in Lemma 3.2 are within $\ell_{\infty} / 2$ of $\ell_{\infty}$ for $z \in \mathbb{S}\left(R_{2}, 1 / 2\right)$, and set $p_{2}^{+}=p_{2}^{-}=R_{2}+i$. For $k$ arbitrary, consider the half-strips $\mathbb{S}(R, 1 / k)$ with $R>R_{k-1}$ and let $p_{k}^{+}$and $p_{k}^{-}$be the two intersection points between the boundary of $\mathbb{S}(R, 1 / k)$ and the boundary of $\mathbb{S}\left(R_{k-1}, 1 /(k-1)\right)$ (The plus being assigned to the point which is above the other one). Again choose $R$ large enough so that three limits in Lemma 3.2 are within $\ell_{\infty} / 2$ of $\ell_{\infty}$ for $z \in \mathbb{S}(R, 1 / k)$. Moreover, choose $R$ so large that the slope of the interval $\left[p_{k-1}^{+}, p_{k}^{+}\right]$is smaller than the slope of the previous interval $\left[p_{k-2}^{+}, p_{k-1}^{+}\right]$, likewise, so that the slope of $\left[p_{k-1}^{-}, p_{k}^{-}\right]$is larger than that of $\left[p_{k-2}^{-}, p_{k-1}^{-}\right]$.

Now let $\Omega$ be the convex hull of the points $p_{k}^{ \pm}, k=2,3, \ldots$ The univalence of $\psi$ on $\Omega$ follows from Proposition 1.10 of [Pom2], because Re $\psi^{\prime}>0$ there and $\Omega$ is convex. Also it follows by construction that $\psi(\Omega)$ contains halfstrips $\mathbb{S}(R, \epsilon)$ of arbitrarily large height. So Corollary 3.4 is proved.

Proof of Theorem 1.14. We proceed as in the uniqueness part of Theorem 1.2 of [PC1]. Suppose $\psi$ and $\tilde{\psi}$ are the two conjugations. Let $\Omega$ and $\tilde{\Omega}$ be the corresponding sets given by Corollary 3.4. Given a small hyperbolic disk centered at $i, \Delta \subset \bar{\Delta} \subset \mathbb{H}$, there is $N$ such that for all $n \geq N$, $\Delta+n b_{0} \in \Omega$, and $\psi\left(\Delta+n b_{0}\right) \subset \tilde{\psi}(\tilde{\Omega})$. So, for $z \in \Delta, z+n b_{0} \in \Omega$ and $\psi\left(z+n b_{0}\right) \in \tilde{\psi}(\tilde{\Omega})$. Let $f$ denote the inverse of $\tilde{\psi}$ on $\tilde{\psi}(\tilde{\Omega})$ which maps $\tilde{\psi}(\tilde{\Omega})$ back to $\tilde{\Omega}$. Then,

$$
\beta(z)=f\left(\psi\left(z+n b_{0}\right)\right)-n \tilde{b}_{0}
$$

is analytic on $\Delta$, one-to-one there, and does not depend on $n \geq N$. In fact, $f\left(\psi\left(z+n b_{0}\right)\right)$ and $f\left(\psi\left(z+n b_{0}+b_{0}\right)\right)$ are mapped by $\tilde{\psi}$ to two points $z_{1}$ and $z_{2}$ such that $\phi\left(z_{2}\right)=z_{1}$. So,

$$
f\left(\psi\left(z+n b_{0}+b_{0}\right)\right)-f\left(\psi\left(z+n b_{0}\right)\right)=\tilde{b}_{0} .
$$


Since the radius of $\Delta$ was arbitrary, this implies that $\beta$ is well-defined and one-to-one on all of $\mathbb{H}$. By interchanging the role of $\psi$ and $\tilde{\psi}$ we find another function $\gamma$ analytic and one-to-one on all of $\mathbb{H}$ which is locally the inverse of $\beta$, and thus also globally. So $\beta$ is an automorphism of $\mathbb{H}$. Since $\beta=$ $\tilde{S}_{n}^{-1} \circ \tilde{\psi}^{-1} \circ \psi \circ S_{n}$, we have $\beta \circ S=\tilde{S} \circ \beta$, i.e., $\beta\left(z+n b_{0}\right)=\beta(z)+n \tilde{b}_{0}$. So $\beta$ fixes infinity and no other point, i.e., $\beta(z)=c_{1} z+b_{1}$ with $b_{1}, c_{1} \in \mathbb{R}$. In particular, $c_{1}\left(z+b_{0}\right)+b_{1}=c_{1} z+b_{1}+\tilde{b}_{0}$, so $c_{1}=\tilde{b}_{0} / b_{0}$. It follows from the definition of $\beta$ that $\tilde{\psi} \circ \beta=\psi$. Hence,

$$
\tilde{\psi}\left(\frac{\tilde{b}_{0}}{b_{0}} z+b_{1}\right)=\psi(z)
$$

With this identity it is easy to check that

$$
\tilde{w}_{n}=\psi\left(z_{1}+n b_{0}\right)
$$

where $z_{1}=\left(b_{0} / \tilde{b}_{0}\right)\left(i-b_{1}\right)$.

Finally, using (3.20) we find that $\ell_{\infty} b_{0}=\tilde{\ell}_{\infty} \tilde{b}_{0}$.

\subsection{Further properties for BISBS of type 1}

Proof of Proposition 1.16. Changing variables from $\mathbb{H}$ to $\mathbb{D}$ via the map $\alpha(z)=(z-i) /(z+i)$, we find that $\Psi=\alpha \circ \psi \circ \alpha^{-1}$ is an analytic self-map of $\mathbb{D}$ with non-tangential limit 1 at 1 , and a calculation shows that

$$
\frac{1-|\Psi(\alpha(z))|^{2}}{1-|\alpha(z)|^{2}}=\frac{\operatorname{Im} \psi(z)}{\operatorname{Im} z} \frac{|z+i|^{2}}{|\psi(z)+i|^{2}}
$$

So when $z=\zeta_{n}$,

$$
\frac{1-\left|\Psi\left(\alpha\left(\zeta_{n}\right)\right)\right|^{2}}{1-\left|\alpha\left(\zeta_{n}\right)\right|^{2}}=y_{n} \frac{4+n^{2} b_{0}^{2}}{x_{n}^{2}+\left(y_{n}+1\right)^{2}}
$$

By (3.21) we find that

$$
\lim _{n \rightarrow \infty} \frac{1-\left|\Psi\left(\alpha\left(\zeta_{n}\right)\right)\right|^{2}}{1-\left|\alpha\left(\zeta_{n}\right)\right|^{2}}=\frac{1}{\ell_{\infty}}
$$

Therefore, by Julia-Carathéodory's Theorem, $\Psi$ has a finite angular derivative at 1 . More specifically, $\Psi^{\prime}(1) \leq 1 / \ell_{\infty}$ (see Theorem $1.2(\mathrm{~d})$ ). Transfering back to $\mathbb{H}$ we find that

$$
\frac{\psi(z)}{z} \longrightarrow A \geq \ell_{\infty}
$$

as $z$ tends to infinity non-tangentially. 
So, by Julia's Lemma, $\psi(z)=A z+q(z)$, where $\operatorname{Im} q(z) \geq 0$ on $\mathbb{H}$ and $q(z) / z$ tends to 0 as $z$ tends to $\infty$ non-tangentially. However,

$$
0 \leq \operatorname{Im} q\left(\zeta_{n}\right)=y_{n}-A \rightarrow \ell_{\infty}-A
$$

So $A=\ell_{\infty}$.

Proof of Corollary 1.17. This follows the same lines argument as the proof of Theorem 2 of [Pom]. By Theorem 1.16, we can write $\psi(z)=\ell_{\infty} z+q(z)$, where $\operatorname{Im} q(z) \geq 0$ and $q(z) / z$ tends to 0 as $z$ tends to $\infty$ non-tangentially. Set $U(z)=\operatorname{Im} q(z) / \operatorname{Im} z$. Then by Schwarz-Pick on $q$, we find

$$
\left|\frac{q(w)-q\left(w^{\prime}\right)}{w-w^{\prime}}\right| \leq \sqrt{U(w) U\left(w^{\prime}\right)}
$$

Let $L=\{t+i, t \geq 0\}$. Then by (3.18), $U(z)$ tends to zero along $L$. On the other hand, from the Poisson integral representation of $\operatorname{Im} q$ it follows that $U$ is a decreasing function of $y$. So given $\varepsilon>0$ there exist $R>0$ such that $U(z)<\varepsilon$ for $z \in Q(R)$. Choose $\varepsilon$ much smaller than $\ell_{\infty}$ and use

$$
\left|\frac{\psi(w)-\psi\left(w^{\prime}\right)}{w-w^{\prime}}\right| \geq \ell_{\infty}-\left|\frac{q(w)-q\left(w^{\prime}\right)}{w-w^{\prime}}\right|
$$

to conclude that $\psi$ is univalent on the corresponding quadrant $Q(R)$.

The similar result about $\phi$ follows the exact same pattern, therefore we omit the details.

Proof of Proposition 1.19. Recall from (3.12) that $H_{n}(z):=\tau_{n}^{-1} \circ \phi \circ$ $\tau_{n+1}(z)$ tends to $z$ uniformly on compact subsets of $\mathbb{H}$. Given a half-strip $\mathbb{S}(R, \epsilon)$, we can find a compact set $K$ such that $\cup_{n=n_{0}}^{\infty} \tau_{n+1}(K)$ covers $\mathbb{S}\left(R^{\prime}, \epsilon\right)$ for some $R^{\prime}>R$ and some $n_{0}$, because $y_{n}$ tends to $\ell_{\infty}$ and $x_{n+1}-x_{n}$ tends to $\ell_{\infty} b_{0}$ (by Theorem 1.14). A computation shows that,

$$
\tau_{n+1}(z)-\phi \circ \tau_{n+1}(z)=y_{n}\left(\tau_{n}^{-1} \circ \tau_{n+1}(z)-H_{n}(z)\right) \rightarrow \ell_{\infty} b_{0}
$$

as $n$ tends to infinity, and this shows the proposition.

\subsection{Non-existence results for parabolic maps of type I}

Proof of Theorem 1.21. Assume $\phi$ is a self-map of $\mathbb{H}$ of parabolic type I. Suppose that $w_{n}=x_{n}+i y_{n}$ is a BISBS. Then, $z_{n}=\phi_{n}\left(w_{0}\right)=u_{n}+i v_{n}$ has non-zero-step, and Pommerenke obtains a conjugation $\sigma$ with range in $\mathbb{H}$, see Theorem 1.8, where $\sigma \circ \phi=\sigma+b$ and $b \neq 0$ is the limit of $\left(u_{n+1}-u_{n}\right) / v_{n}$, see (1.6). By Remark 1 of [Pom], $\operatorname{Arg} z_{n}$ either tends to 0 or to $\pi$. Let us 
assume without loss of generality that it tends to $\pi$, so that $b<0$. Then, there exist $n_{0}$ so that for $n \geq n_{0}, u_{n}-u_{n+1}$ is positive, and greater than $(|b| / 2) v_{n}$. By telescoping sums

$$
\left|u_{n}\right| \geq(|b| / 2) \sum_{k=n_{0}}^{n-1} v_{k}+\left|u_{n_{0}}\right| \geq(|b| / 2) v_{n_{0}}\left(n-n_{0}\right)+\left|u_{n_{0}}\right|
$$

In particular,

$$
\liminf _{n \rightarrow \infty} \frac{\left|u_{n}\right|}{n} \geq(|b| / 2) v_{n_{0}}
$$

This implies that we always have $\liminf \left|u_{n}\right| / n>0$, and $\lim \left|u_{n}\right| / n=+\infty$ when $v_{n} \uparrow \infty$.

On the other hand, assume first that $\operatorname{Arg} w_{n}$ tends to 0. Then, $\left(x_{n+1}-\right.$ $\left.x_{n}\right) / y_{n}$ tends to $b_{0}>0$, see (1.9). So there exists $n_{1}$ such that for $n \geq n_{1}$, $x_{n+1}-x_{n}$ is less than $2 b_{0} y_{n}$, and therefore

$$
x_{n} \leq 2 b_{0} y_{n_{1}}\left(n-n_{1}\right)+x_{n_{1}}
$$

A similar estimate can be obtained if $\operatorname{Arg} w_{n}$ tends to $\pi$. So

$$
\limsup _{n \rightarrow \infty} \frac{\left|x_{n}\right|}{n} \leq 2 b_{0} y_{n_{1}}
$$

This implies that we always have $\lim \sup \left|x_{n}\right| / n<\infty$, and $\lim \left|x_{n}\right| / n=0$ when $y_{n} \downarrow 0$.

Now, since $\sigma$ is a self-map of $\mathbb{H}, d\left(\sigma\left(w_{0}\right), \sigma\left(z_{n}\right)\right) \leq d\left(w_{0}, z_{n}\right)$ and the fact that $\sigma\left(z_{n}\right)=\sigma\left(w_{0}\right)+n b$ imply that

$$
\frac{v_{n} y_{0}}{\left(u_{n}-x_{0}\right)^{2}+\left(v_{n}+y_{0}\right)^{2}} \leq \frac{\left(\operatorname{Im} \sigma\left(w_{0}\right)\right)^{2}}{n^{2} b^{2}+4\left(\operatorname{Im} \sigma\left(w_{0}\right)\right)^{2}}
$$

Now $v_{n} / u_{n}$ tends to zero, because $\operatorname{Arg} z_{n}$ tends to $\pi$. So

$$
\frac{v_{n}}{u_{n}^{2}} \leq \frac{C_{1}}{n^{2}}
$$

for some constant $C_{1}>0$. On the other hand, let $\psi$ be the conjugation provided by Theorem 1.12. The fact that $\psi\left(i-n b_{0}\right)=z_{n}$ and SchwarzPick's inequality $d\left(z_{n}, w_{n}\right) \leq d\left(i-n b_{0}, i+n b_{0}\right)$ imply that

$$
\frac{1}{4 n^{2} b_{0}^{2}+4} \leq \frac{v_{n} y_{n}}{\left(x_{n}-u_{n}\right)^{2}+\left(v_{n}+y_{n}\right)^{2}}
$$


So that by (3.24),

$$
y_{n} \geq \frac{C_{2}}{n^{2}} \frac{\left(x_{n}-u_{n}\right)^{2}}{v_{n}} \geq \frac{C_{2}}{C_{1}}\left(\frac{x_{n}}{u_{n}}-1\right)^{2}
$$

for some constant $C_{2}>0$.

Assume now that $w_{n}$ is of type 2, i.e. $y_{n} \downarrow 0$. Then (3.25) yields

$$
\frac{x_{n}}{u_{n}} \longrightarrow 1 \text {. }
$$

But this contradicts the fact that, in this case,

$$
\lim \left|x_{n}\right| / n=0 \quad \text { and } \quad \liminf \left|u_{n}\right| / n>0 .
$$

Proof of Corollary 1.22. Consider Pommerenke's map $\sigma$ (see Th. 1.8), in the case when $b \neq 0$ and the imaginary parts of the forward iterates remain bounded above (type Ia). Assume that $z_{n}=\phi_{n}(i)=u_{n}+i v_{n}$ and $\operatorname{Arg} z_{n}$ tends to 0 , so that $b>0$. By Lemma 2 of [Pom], $\sigma\left(u_{n}+z v_{n}\right)-n b$ tends to $z$ uniformly on compact subsets of $\mathbb{H}$. This can be used to show that

$$
\text { lat. }-\lim _{z \rightarrow+\infty} \operatorname{Re} \sigma(z)=+\infty
$$

Suppose now that $\phi$ has a BISBS whose argument tends to 0 as well. By Theorem 1.21 such BISBS would have to be of type 1 . So it eventually belongs to some half-strip $\mathbb{S}(R, \epsilon)$. But $\sigma\left(w_{n}\right)=\sigma\left(w_{0}\right)-n b$, so $\operatorname{Re} \sigma$ tends to $-\infty$ along $w_{n}$, and this contradicts (3.27).

\section{Examples}

All of the examples that follow are obtained by conjugating $\phi$ to a translation on an appropriately chosen simply connected region, so that the resulting Riemann map, which does the conjugation, can be written down explicitly.

\section{Type Ia $\emptyset$ (non-zero-step/finite-height/no-BISBS):}

$$
\phi(z)=\left[\sqrt{(\sqrt{z}+1)^{2}+1}-1\right]^{2}
$$

Here $\phi$ is conjugated to translation by 1 on the image of $\mathbb{H}$ under the conformal map $(\sqrt{z}+1)^{2}$. Then

$$
z_{n}=(\sqrt{n+i}-1)^{2}=n-1+i-2 \sqrt{n+i}
$$

is a forward-iteration sequence, and there are no BISBS. 
Type Ib $\emptyset$ (non-zero-step/infinite-height/no-BISBS):

$$
\phi(z)=(\sqrt{z}+1)^{2}
$$

Here $\phi$ is conjugated to translation by 1 on the image of $\mathbb{H}$ under the conformal map $\sqrt{z}$. Then

$$
z_{n}=(n+i)^{2}=n^{2}-1+2 i n
$$

is a forward-iteration sequence, and there are no BISBS. Note that $z_{n} / v_{n}=$ $(n-1 / n)+2 i$ so it is non-zero-step.

Type IIb $\emptyset$ (zero-step/infinite-height/no-BISBS):

$$
\phi(z)=z+i
$$

Type Ia1 (non-zero-step/finite-height with a BISBS of non-zeroheight):

$$
\phi(z)=z+1
$$

Type IIb1 (zero-step/infinite-height with a BISBS of non-zeroheight):

$$
\phi(z)=\left(\sqrt{(\sqrt{z}-1)^{2}-1}+1\right)^{2}
$$

Here $\phi$ is conjugated to translation by -1 on the image of $\mathbb{H}$ under the conformal map $(\sqrt{z}-1)^{2}$. Then

$$
z_{n}=(1+i \sqrt{n})^{2}=1-n+2 i \sqrt{n}
$$

is a forward-iteration sequence, $z_{n} / v_{n}=-\sqrt{n}+1 / \sqrt{n}+2 i$ so the step goes to zero. Moreover,

$$
w_{n}=(\sqrt{n+i}+1)^{2}=n+1+i+2 \sqrt{n+i}
$$

is a BISBS of non-zero-height.

Type IIb2 (zero-step/infinite-height with a BISBS of zero-height):

$$
\phi(z)=\sqrt{z^{2}-1}
$$

Here $\phi$ is conjugated to translation by -1 on the image of $\mathbb{H}$ under the conformal map $z^{2}$. Then

$$
z_{n}=i \sqrt{n}
$$

is a forward orbit, and

$$
w_{n}=\sqrt{n+i}
$$

is a BISBS of zero-height.

Examples of Ib1, IIa1, IIa2 types are still missing. 


\section{References}

[BP] Baker, I., Pommerenke, C.: On the iteration of analytic functions in a halfplane II. J. London Math. Soc. (2) 20 (1979), 255-258.

[Br] BraCCI, F.: Fixed points of commuting holomorphic mappings other than the Wolff point. Trans. Amer. Math. Soc. 355 (2003), no. 6, 25692584 .

[CM] Cowen, C., MacCluer, B.: Composition operators on spaces of analytic functions. Studies in Advanced Mathematics, CRC Press, Boca Raton, FL, 1995.

[CP] Cowen, C., Pommerenke, C.: Inequalities for the angular derivative of an analytic function in the unit disk. J. London Math. Soc. (2) 26 (1982), 271-289.

[PC1] Poggi-Corradini, P.: Canonical conjugations at fixed points other than the Denjoy-Wolff point. Ann. Acad. Sci. Fenn. Math. 25 (2000), 487-499.

[PC2] Poggi-Corradini, P.: Angular derivatives at boundary fixed points for self-maps of the disk. Proc. Amer. Math. Soc. 126 (1998), no. 6, $1697-1708$.

[Pom] Pommerenke, C.: On the iteration of analytic functions in a halfplane, I. J. London Math. Soc. (2) 19 (1979), 439-447.

[Pom2] Pommerenke, C.: Boundary behaviour of conformal maps. Grundlehren der Mathematischen Wissenschaften 299. Springer-Verlag, Berlin, 1992.

[Sh] Shapiro, J.: Composition operators and classical function theory. Universitext: Tracts in Mathematics. Springer-Verlag, New York, 1993.

Recibido: 10 de septiembre de 2001

Pietro Poggi-Corradini Department of Mathematics Cardwell Hall, Kansas State University Manhattan, KS 66506, USA pietro@math.ksu.edu 